\title{
Correlation between peak nasal inspiratory flow and peak expiratory flow in children and adolescents*
}

\author{
Cinthia Chaves ${ }^{1}$ Cássio da Cunha Ibiapina', Cláudia Ribeiro de Andrade', \\ Ricardo Godinho', Cristina Gonçalves Alvim', Álvaro Augusto Cruz² \\ Rhinology 50: 381-385, 2012 \\ DOl:10.4193/Rhino12.073 \\ Paediatric Pulmonology Unit, University Hospital, Federal University of Minas Gerais, Belo Horizonte, Brazil \\ *Received for publication: \\ ProAR, Faculdade de Medicina da Bahia, Universidade Federal de Minas Gerais, Belo Horizonte, Brazil \\ May 4, 2012 \\ Accepted: August 10, 2012
}

\section{SUMMARY}

Background: Peak nasal inspiratory flow (PNIF) has been proposed as a simple method to evaluate nasal patency. Asthma and allergic rhinitis are commonly associated, and lower airway assessment can provide information concerning an objective interpretation of nasal function.

Aims: To determine whether the PNIF is correlated with peak expiratory flow (PEF) in children and adolescents.

Methods and Results: Cross-sectional study carried out in healthy students randomly chosen in 14 public schools of the city of Belo Horizonte. PNIF and PEF were assessed for each subject as the following characteristics: gender, height, weight and age. We created a linear regression model to explain the PNIF, in which we included all the variables with a $p$ value $\leq 0.25$ in a univariate analysis, and to calculate the relationship between the maximum PNIF and maximum PEF by the Spearman correlation coefficient. In total, 297 healthy subjects, aged between six and eighteen years were evaluated. A positive and significant correlation between PNIF and PEF was found.

Conclusions: PEF is predictive of PNIF. However, these measures evaluate two distinct segments of the airways and should be both obtained for a more precise assessment of airflow limitation.

Key words: nose, lungs, respiratory function tests, peak expiratory flow, peak nasal inspiratory flow

\section{Introduction}

Asthma is considered one of the major chronic diseases of the world ${ }^{(1)}$, and an important cause of childhood morbidity. Allergic rhinitis (AR) is also a global public health problem, and its prevalence is estimated to be at least 10 to $25 \%$ in the world population. The literature has stressed the hypothesis that asthma and allergic rhinitis are manifestations of the same disease, which affects the entire respiratory tract ${ }^{(2,3)}$. In many instances, uncontrolled rhinitis may add to the unsatisfactory control of asthma, or it may even be a sign of a severe disease of the respiratory tract.

Rhinitis and asthma represent a large burden to health care systems, and rhinitis increases the cost of asthma ${ }^{(3,4)}$.

As asthma and allergic rhinitis usually happen concurrently, the evidence collected by Allergic Rhinitis and its impact on Asthma (ARIA) initiative is being broadly disseminated, aiming for increased implementation and provision of best care for patients with this often neglected disease of the airways ${ }^{(5,6)}$.

Asthma diagnosis is frequently ratified by pulmonary function tests, broadly utilized in medical practice ${ }^{(7)}$. On the other hand, nasal patency assessment with objective measures is still not a part of clinical routine tests ${ }^{(8)}$. PNIF has been deemed a 
simple and quick alternative, which can be easily learned and interpreted ${ }^{(9,10)}$. It is a well studied technique, and it bears basal parameters of normality specified for adults of different ethnical backgrounds ${ }^{(11-13)}$ and also for the pediatric population ${ }^{(14-16,17)}$.

With the goal of determining whether the PEF value is a determinant of the PNIF, a study led by Ottaviano et al., in a sample made up of adults, concluded that the PEF value is predictive of the PNIF ${ }^{(18)}$

Nonetheless, this was not found in studies with children assessing the two tests simultaneously. The goal of the present study is to determine whether PNIF is correlated with the PEF value in healthy children and adolescents.

\section{Materials and methods}

This is a cross-sectional study carried out in 14 public schools of the city of Belo Horizonte, Minas Gerais state, Brazil. Healthy children and adolescents between six and eighteen years of age were randomly chosen from different ethnic backgrounds.

Inclusion and exclusion criteria

School children and adolescents with negative answers in the International Study of Asthma and Allergies in Childhood (ISAAC) ${ }^{(19)}$ questionnaire were included.

Children and adolescents with a positive answer in the ISAAC questionnaire regarding the report of sneezes, runny nose, nasal obstruction, wheezing or whistling in the past 12 months - concerning allergic rhinitis or asthma were excluded. Those with moderate to severe adenoid hypertrophy, nasal septum deviation, nasal polyps and upper airway infections - diagnosed in the physical exam carried out by a physician, or those unable to perform the maneuvers required to measure PNIF and PEF were also excluded.

\section{Procedures}

Tests maneuvers were executed under the researchers' supervision. The ISAAC questionnaires were filled out by teenagers older than 12 years of age. For those younger than 12 years of age, the questionnaires were filled out by their parents/guardians. It was also collected data regarding gender, age, weight and height.

\section{Measurements}

PNIF. Before checking the PNIF, the subjects cleaned their noses - mildly blowing their noses to clear up any nasal secretion. The facial mask was carefully applied and the subjects were instructed to do a nasal inspiration with their mouths closed and, from the residual volume, to reach full pulmonary capacity. The equipment utilized was the In-check-inspiratory flow meter (Clement Clarke, Harlow, UK, 50 to 300 L/m). It was carried out at least three measurements. The highest value was used for the analysis. All the measures were taken with the subject standing up.
PEF. The PEF measurement was carried out using the Mini-Wright Peak Expiratory Flow Meter (Clement Clarke, UK, 60 to 800 L/m), with the child/adolescent standing up, previously instructed to reach the maximum flow during forced expiration. For the analysis, it was chosen the highest individual value from three consecutive takings.

\section{Statistics}

The sample size was estimated based on comparing the PNIF mean values among the male and female subjects. Considering $5 \%$ of significance and a power of $80 \%$, it would be necessary to have 126 subjects from each gender, making up a total of 252 cases.

For the descriptive analysis it was calculated the frequencies and percentages of the different categorical variables, the central trend measures (mean and median); and the standard deviation. The maximum PNIF were compared with age, height and weight based on the calculations of the Spearman correlation coefficients. The Mann-Whitney test compared the response variable and gender. Then, a linear regression model was used to explain the PNIF, in which the initial model included all the characteristics with a $p$-value $\leq 0.25$ in the univariate analysis. The final model was the one including the variables with statistical significance ( $p$ value $\leq 0.05$ ).

The Spearman correlation coefficient assessed the correlation between the maximum PNIF and the maximum PEF, since the normality assumption - checked by the Shapiro-Wilk test - was violated.

\section{Ethics}

The study protocol and the written informed consent were approved by the Committee of Ethics in Human Research of the Federal University of Minas Gerais. The protocol number of the study given by the Ethical Committee: $n^{\circ}$ ETIC 584/08.

\section{Results}

The sample population was composed by 297 healthy children and adolescents, aged between six and 18 years old. Their height, weight, $Z$ scores and percentiles are described in Table 1.

Among the 297 participants, $54.2 \%$ were females. The highest descriptions among the three measurements made for PNIF and $\mathrm{PEF}$, on average, was $105 \mathrm{~L} / \mathrm{min}$ and $300 \mathrm{~L} / \mathrm{min}$, respectively. The results on PNIF and PEF are presented in Table 2.

The female subjects had a mean PNIF of $101.5 \mathrm{~L} / \mathrm{min}$ and males had $109.3 \mathrm{~L} / \mathrm{min}$, with a $\mathrm{p}$-value of 0.027 .

We analyzed the correlations between the PNIF and the quantitative variables: height (centimeters), age (months), weight (kilograms), Z score weight/age, Z score body mass index (BMI), 
Table 1. Height, age, weight, Z scores and percentiles.

\begin{tabular}{lcccc}
\multicolumn{1}{c}{ Characteristics } & Mean & S.D. & Min & Max \\
Height (cm) & 151.7 & 14.6 & 115.0 & 188.0 \\
Age (years) & 12.2 & 2.7 & 6.0 & 17.6 \\
Weight (kg) & 43.5 & 14.2 & 17.0 & 91.0 \\
Score Z & & & & \\
Weight/Age & 0.3 & 6.8 & -18.7 & 102.3 \\
Height/Age & 0.4 & 3.0 & -7.1 & 39.4 \\
BMI & -0.2 & 1.2 & -5.3 & 2.3 \\
Percentile & & & & \\
Weight/Age & 49.0 & 30.8 & 0.0 & 100.0 \\
Height/Age & 55.9 & 29.6 & 0.0 & 100.0 \\
BMI & 44.7 & 31.5 & 0.0 & 99.0
\end{tabular}

S.D. $=$ standard deviation

Table 2. PNIF and PEF results in healthy children and adolescents.

\begin{tabular}{|lcccc}
\hline \multicolumn{1}{|c}{ Variables } & Mean & S.D. & Min. & Max. \\
\hline PNIF & 91.12 & 32.6 & 30.00 & 200.00 \\
\hline 1st measurement & 95.56 & 34.8 & 30.00 & 250.00 \\
\hline 2nd measurement & 97.73 & 35.5 & 35.00 & 250.00 \\
\hline 3rd measurement & 105.20 & 35.1 & 40.00 & 250.00 \\
\hline Maximum & & & & \\
\hline PEF & 265.17 & 80.2 & 90.00 & 560.00 \\
\hline 1st measurement & 282.95 & 84.5 & 100.00 & 620.00 \\
\hline 2nd measurement & 288.27 & 85.8 & 110.00 & 670.00 \\
\hline 3rd measurement & 300.30 & 88.1 & 120.00 & 670.00 \\
\hline Maximum & & & & \\
\hline
\end{tabular}

Values in $\mathrm{L} / \mathrm{m}$.

weight/age percentile; height/age percentile and BMI percentile and data are shown in Table 3.

Maximum PNIF was compared to height, age, weight, Z scores and percentiles using the Spearman's correlation coefficients. To compare the response variable and gender, the Mann-Whitney test was used. Then, we developed a linear regression model to explain the PNIF. All the variables mentioned were included in the multivariate model adjustment process.

The final model included only those variables with statistical significance ( $p$ value $\leq 0.05$ ).

Five multivariate linear regression models were adjusted for the PNIF variable. The the following clinically variables were used: gender, age, height/age percentile and the data are presented on Table 4. 
Table 3. Comparison of the quantitative variables with PNIF.

\begin{tabular}{|c|c|c|}
\hline Variables & Correlation coefficient & p value \\
\hline Height (cm) & 0.330 & $<0.001^{1}$ \\
\hline Age (months) & 0.217 & $<0.001^{1}$ \\
\hline Weight (kg) & 0.300 & $<0.001^{1}$ \\
\hline \multicolumn{3}{|l|}{ Z score } \\
\hline Weight/Age & 0.136 & $0.019^{1}$ \\
\hline Height/Age & 0.167 & $0.004^{1}$ \\
\hline BMI & 0.074 & $0.203^{1}$ \\
\hline \multicolumn{3}{|l|}{ Percentile } \\
\hline Weight/Age & 0.136 & $0.019^{1}$ \\
\hline Height/Age & 0.167 & $0.004^{1}$ \\
\hline BMI & 0.074 & $0.203^{1}$ \\
\hline
\end{tabular}

'Spearman's correlation coefficient.

Table 4. Linear regression model for the transformed PNIF variable - gender, age and height/age percentile.

\begin{tabular}{|c|c|c|c|c|c|}
\hline \multirow[t]{2}{*}{ Model } & \multirow[t]{2}{*}{ Coefficient } & \multirow[t]{2}{*}{ Standard Error } & \multirow[t]{2}{*}{ p value } & \multicolumn{2}{|c|}{$\mathrm{Cl} 95 \%$} \\
\hline & & & & Lower & Upper \\
\hline Constant & 7.57 & 0.50 & $<0.001$ & & \\
\hline \multicolumn{6}{|l|}{ Gender } \\
\hline Male & 0.41 & 0.19 & 0.032 & 0.04 & 0.78 \\
\hline \multicolumn{6}{|l|}{ Female } \\
\hline Age (months) & 0.01 & 0.002 & $<0.001$ & 0.006 & 0.02 \\
\hline Height/age percentile & 0.01 & 0.003 & $<0.001$ & 0.006 & 0.02 \\
\hline
\end{tabular}

$\mathrm{Cl}$ : Confidence interval.

Thus, with increasing age and height/age percentile, there is a PNIF increase. The male subjects had a higher PNIF. The correlation shown in Figure 1 was positive and significant $(r=0.433)$.

\section{Discussion}

Considering the relationship between asthma and allergic rhinitis ${ }^{(20,21)}$, associated to estimates of their raise in incidence in some parts of the world ${ }^{(1)}$, researchers are encouraged to find strategies to face it, and drive new research to better understand and control it.

Objective nasal patency assessment tests may add relevant information about nasal function and are valuable to improve handling of upper airway disorders ${ }^{(22)}$. Such usefulness happens mainly among children, which objective measurements are even more relevant because of the peculiarity of the subjective information, which are, frequently, provided by parents or guardians ${ }^{(23)}$
Despite PNIF's practicality and applicability, such a test still requires further investigation ${ }^{(24)}$. The study about the relationship between its values and PEF is especially interesting, since it translates the current thinking of a single airway disease and, in such context, the need for a critical analysis of the airways, in an integrated fashion, to have a more reliable decision concerning nasal patency. The results are useful in clinical practice and the physicians could explore routinely both tests to better understand the global airway conditions.

The attention given to the study of the PNIF and PEF correlation is still very new. In a prior study, made up of 100 subjects aged between 15 and 71 years, without a past of smoking, otorhinolaryngological surgeries, symptoms of asthma, nasal obstruction or other respiratory symptoms, Ottaviano et al. analyzed the correlation between the PNIF and different covariables, including PEF, and reported a positive correlation $(r=0.263)^{(18)}$. 


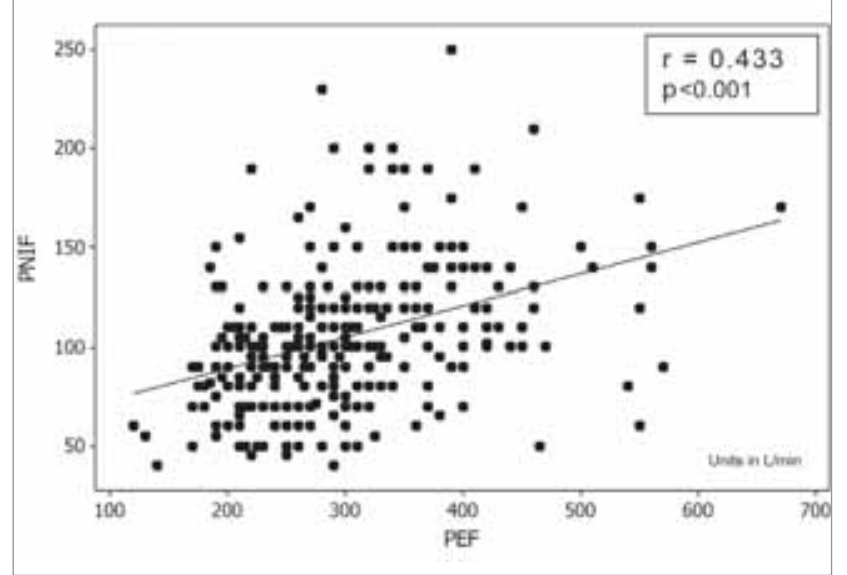

Figure 1. Correlation between PEF and PNIF.
The present investigation involved 297 healthy children and adolescents between 6 and 18 years of age. A positive correlation between PNIF and gender, age, height/weight percentile and PEF was found. Thus, as age and the height/age percentile increases, PNIF also increases. There is also a moderate correlation between PNIF and PEF ( $r=0.433 ; p \leq 0.001)$. In conclusion, the PEF is predictive and informative of the PNIF value in healthy children.

As expected and already demonstrated in adults, low values of PNIF must be confirmed by PEF values, because they may reflect a reduction in lower airway patency. Therefore, the results suggest that the two methods must be, ideally, utilized together in the daily practice of health care professionals.

\section{Conflict of interest statement}

There are no competing interests for any of the authors.

\section{REFERENCES}

1. Anandan C, Nurmatov U, van Schayck OC, Sheikh A. Is the prevalence of asthma declining? Systematic review of epidemiological studies. Allergy. 2010; 65: 152-167.

2. Togias A. Rhinitis and asthma: evidence for respiratory system integration. J Allergy Clin Immunol. 2003; 111:1171-83; quiz 1184

3. Cruz AA, Popov T, Pawankar R, et al. ARIA Initiative Scientific Committee. Common characteristics of upper and lower airways in rhinitis and asthma: ARIA update, in collaboration with GA(2)LEN. Allergy. 2007; 62 Suppl 84: 1-41.

4. Cruz AA. The "united airways" require an holistic approach to management. Allergy. 2005; 60: 871-874.

5. Kupczyk M, Haahtela T, Cruz AA, Kuna P. Reduction of asthma burden is possible through National Asthma. Allergy. 2010; 65: 415-419.

6. J. Bousquet1, N. Khaltaev, A. A. Cruz, et al. Allergic Rhinitis and its Impact on Asthma (ARIA) 2008 Update. Allergy. 2008: 63 (Suppl. 86): 8-160.

7. Bousquet J, Clark TJ, Hurd S, et al. GINA guidelines on asthma and beyond. Allergy. 2007; 62: 102-112.

8. Eccles R. Is a normal range of nasal function beyond the reach of rhinologists? Am J Rhinol. 2006; 20: 205.

9. Timperley D, Srubisky A, Stow N, Marcells GN, Harvey RJ. Minimal clinically important differences in nasal peak inspiratory flow. Rhinology. 2011; 49: 37-40.

10. Cho SI, Hauser R, Christiani DCChest. Reproducibility of nasal peak inspiratory flow among healthy adults: assessment of epidemiologic utility. Chest. 1997; 112: 15471553

11. Klossek JM, Lebreton JP, Delagranda A, Dufour X. PNIF measurement in a healthy French population. A prospective study about 234 patients. Rhinology. 2009; 47: 389-392.

12. Bouzgarou MD, Saad HB, Chouchane $A$, Cheikh IB, Zbidi A. North African reference equation for peak nasal inspiratory flow. J Laryngol Otol. 2011; 28: 1-8.

13. Ottaviano G, Scadding GK, Coles S, Lund VJ. Peak nasal inspiratory flow; normal range in adult population. Rhinology. 2006; 44: 32-35.

14. Prescott CA, Prescott KE. Peak nasal inspiratory flow measurement: an investigation in children. Int J Pediatr Otorhinolaryngol. 1995; 32: 137-141

15. Papachristou A, Bourli E, Aivazi D, et al. Normal peak nasal inspiratory flow rate values in Greek children and adolescents. Hippokratia. 2008; 12: 94-97.

16. Ibiapina CC, Andrade CR, Camargos PA Alvim CG, Cruz AA. Reference values for peak nasal inspiratory flow in children and adolescents in Brazil. Rhinology. 2011; 49: 304-308.

17. Van Spronsen E, Ebbens FA, Fokkens WJ Normal peak nasal inspiratory flow rate values in healthy children aged 6 to 11 years in the Netherlands. Rhinology. 2012; 50: 21-25.

18. Ottaviano G, Lund VJ, Coles S, Staffieri A Scadding GK. Does peak nasal inspiratory flow relate to peak expiratory flow? Rhinology. 2008; 46: 200-203.

19. Isaac. Isaac Manual (2nd Edition). Auckland/ Münster. December 1993.

20. Leynaert B, Neukirch C, Liard R, Bousquet J, Neukirch F. Quality of life in allergic rhinitis and asthma. A population-based study of young adults. Am J Respir Crit Care Med. 2000; 162 (4 Pt 1): 1391-1396.

21. Bachert C, van Cauwenberge P, Khaltaev N World Health Organization. Allergic rhinitis and its impact on asthma. In collaboration with the World Health Organization. Executive summary of the workshop report. 7-10 December 1999, Geneva, Switzerland.
Allergy. 2002; 57: 841-855.

22. Eccles R. A guide to practical aspects of measurement of human nasal airflow by rhinomanometry. Rhinology. 2011; 49: 2-10.

23. Gomes D de L, Camargos PA, Ibiapina C da $C$, de Andrade CR. Nasal peak inspiratory flow and clinical score in children and adolescents with allergic rhinitis. Rhinology 2008; 46: 276-280.

24. Clarke RW, Jones AS. The limitations of peak nasal flow measurement. Clin. Otolaryngol Allied Sci. 1994; 19: 502-504.

Cássio da Cunha Ibiapina

Paediatric Pulmonology Unit, University Hospital

Federal University of Minas Gerais Avenida Professor Alfredo Balena

$190 /$

Room 267

30130-100 Belo Horizonte

Brazil

Tel: +55-31-3409 9772

Fax: +55-31-3409 9664

E-mail: cassioibiapina@terra.com.br 